\title{
CONVERTER WITH SYNCHRONOUS RECTIFIERS FOR ELECTROPLATING
}

This paper presents the requirements for resources for electroplating processes, principles of the synchronous rectifier and its control. It describes simulation and experimental verification of the synchronous rectifier, compares the efficiency of switching power supply using a synchronous rectifier and a diode rectifier, where body diode of MOSFET transistor is used for rectification. Finally the paper brings thermal images of synchronous rectifier transistors.

\section{Introduction}

Switching power supplies for electroplating processes are characterized by low output voltage (tens of volts), which is comparable to the voltage drop of rectifying elements and large current output (thousands of amps). Permissible output current ripple is $2-5 \%$ of the average current. Ripple of the output voltage is $1 \%$. Significant losses are caused by current flow through semiconductor structure for required parameters.

The goal of research is minimizing switching power supply losses using synchronous rectifier. It is necessary to use a transistor with low resistance in the conducting state of structure. It is also necessary to select an appropriate topology of the synchronous rectifier circuit with respect to the number of transistors. Very important part of topology is control of MOSFET transistors, which enables the transistor to conduct current from zero voltage UDS. Commutation failure is not acceptable. The article describes the issue of power supplies for electroplating processes, their activities and various topologies; control and principle of synchronous rectifier. It also brings experimental verification of the synchronous rectifier, efficiency comparison of switching power supply using a synchronous rectifier and a diode rectifier, where body diode of MOSFET transistor is used for rectification [1].

\section{Requirements for Power Supply}

All technological processes take place in an environment of galvanic baths of various sizes and designs. Their activities require (with some exceptions) power supplies of small DC voltage with high output currents. In the aspect of required performance we can divide resources for electrochemical applications to several cate- gories. Power supplies with minimal performance (up to $1 \mathrm{kVA}$ ) are less important and used for technological processes as degreasing e.g. Medium performance (up to $40 \mathrm{kVA}$ ) is typical for the metallization and small refinery links and big performance supplies (from $100 \mathrm{kVA}$ ) are used for large refining links and production of chlorine and aluminum.

Supplies specific characteristics depend on the character of electroplating techniques. It is a particular need for low output voltage, very high currents. The fact that the bath is a source of reverse voltage of variable level is important, too. For some technological processes, it is also necessary to allow change the polarity of the output voltage of power supply. Output current with minimal ripple allows achieve superior surface in most cases, i.e. homogeneous layer of deposited metal. In the case of minimal current ripple of metalized material surface, liquid products are removed worse and we must remove it with additive surface washing. The optimal value of current ripple is topic of debates for years, but acceptable level of current ripple is set to $2-5 \%$ of current average value [1]

\section{Synchronous Rectifier}

In synchronous rectifier, diodes are replaced by MOSFET transistors. Diodes must be engaged with respect to the fact that in presence of driving signal in the gate transistor works in the third quadrant of the static output characteristics (Fig. 1). In the third quadrant, the body diode is conductive, because it is polarized in forward direction. If the supply output voltage is polarized reverse, transistor works in the first quadrant of its static characteristics and transistor is closed. We can also see that the body diode is in the blocking state. MOSFET transistors are able to conduct current at zero voltage $U_{D S}$. This is not able in the diode due to

\footnotetext{
* Peter Hurtuk, Martin Priecinsky, Anna Kondelova

Department of Mechatronics and Electronics, Faculty of Electrical Engineering, University of Zilina, Slovakia, E-mail: hurtuk@fel.utc.sk
} 
its threshold voltage value. Threshold diode voltage in its conductive state causes loss proportional to the product $U_{T H} \cdot I_{F(A V)}$. In synchronous rectification this part of the losses is eliminated.

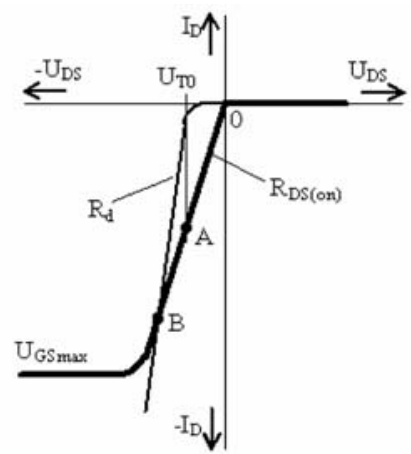

Fig. 1 V-A characteristic of MOSFET transistor and the body diode

If the transistor is open and operates in the third quadrant of its output characteristics, it can conduct current from the zero voltage without applying the threshold voltage of the body diode. Voltage drop on $R_{D S(o n)}$ increases with increasing current; in point A this drop reaches the body diode threshold voltage value, the diode opens and starts to conduct current. In this time, both elements are conductive simultaneously as parallel elements and hence the total resistance of MOSFET transistor is formed by parallel connecting $R_{D S(o n)}$ and dynamic resistance $R_{d}$ of body diode (Fig 2 ). Increasing voltage on structure (from zero to the threshold voltage of body diode) has characteristic slope of $R_{D S(o n)}$. Next increasing of voltage has characteristic slope of parallel connected $R_{D S(o n)}$ and $R_{d}$. Values of current depend on a value of voltage drop on transistor and body diode. In point $\mathrm{B}$, both currents are equal. In next increasing of voltage higher current is flowing through diode than through the transistor [2] and [3].

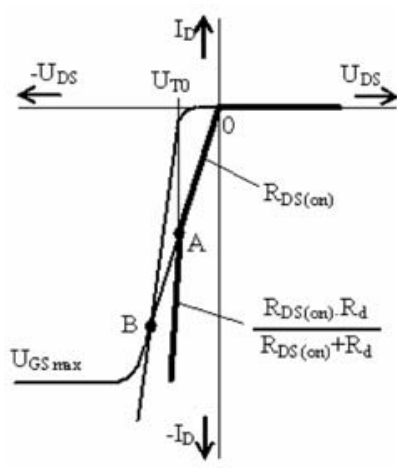

Fig. 2 The resulting output characteristics structure

Power losses of the MOSFET transistor for voltage drops from zero to the value of body diode threshold voltage are expressed in equation 1; losses from A to B are expressed in equation 2 . These losses represent a voltage drop of synchronous rectifier:

$$
\begin{aligned}
& \Delta P_{1}=R_{D S(o n)} \cdot I_{D}^{2}, \\
& \Delta P_{2}=\frac{R_{D S(o n)} \cdot R_{d}}{R_{D S(o n)}+R_{d}} \cdot I_{D}^{2} .
\end{aligned}
$$

The diode is characterized with permanent voltage drop due to its threshold voltage and the voltage drop due to the dynamic resistance $R_{d}$. Power losses of diode are expressed in equation 3 :

$$
\Delta P_{3}=U_{T 0} \cdot I_{D}+R_{d} \cdot I_{D}^{2} .
$$

Fig. 3 shows a comparison of conduction losses for synchronous and diode rectifier.

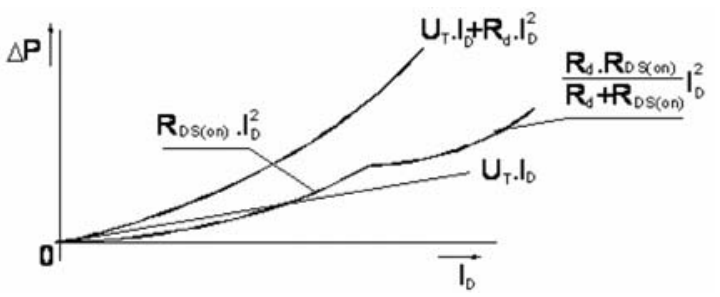

Fig. 3 Comparison of conduction losses

In addition to conduction losses, in semi-conductive structures we can calculate switching losses, control losses and loss in the rectifier commutation. These losses depend on the topology of the rectifier circuit. The energy required for charging and discharging of MOSFET input capacity generates control losses and they are increasing with increasing frequency.

\section{Synchronous Rectifier Control}

Basic topologies of synchronous rectifiers are derived from conventional ones. There are two types of control, external control and self-control of synchronous rectifier transistors.

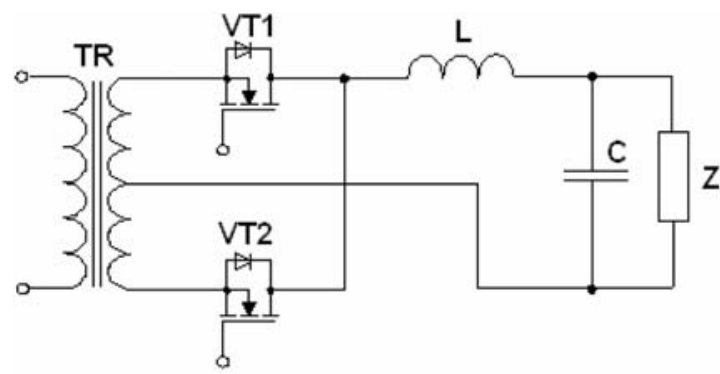

Fig. 4 Control synchronous rectifier

An example of external control is in Fig. 4. Control of synchronous rectifier MOSFET transistor contains control and driver circuit, which generates control signal. We must set precise timing of control signals, which is the most critical point of this work. 
Timing of control signals can be obtained by two ways. The first is to obtain control data from the primary site of converter, from control circuit of the converter. Galvanic isolation of control signal in the secondary side of converter from the primary one is needed. Precise timing of control signals to the gates of MOSFET is important.

Additional control losses occur in the external control. The advantage of the external control is non-dependency of control signals due to load variations, simple gate protection and particularly accurate timing of control signals.

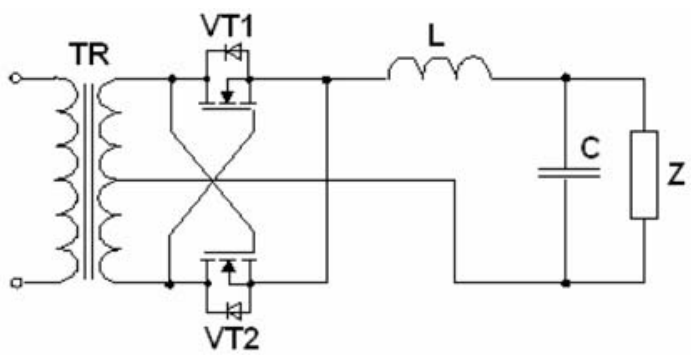

Fig. 5 Self-driven synchronous rectifier

In self-driven synchronous rectifiers voltage from secondary transformer side is directly used for transistor control. The selfdriven synchronous rectifier is shown in Fig. 5. With the power supply topology on the primary side of transformer the shape control curves are changing. Certain requirements are placed on the control curve. The voltage levels must be high enough for safe switching of transistors, but not too high because of the security of the gates. Energy for control used to charge the parasitic capacity of MOSFET transistors can be used from the magnetizing or dispersion transformer inductance [4] and [5].

\section{Simulation Analysis Switching Power Supply}

Simulation analysis is used for verification of the networks operation. With the changing of circuit parameters, the values of its variables $(\mathrm{V}, \mathrm{I}, \mathrm{W})$ are checked. The most important value is power dissipations on transistor without control signal, where only the inner diode conduct the current, and with control of this transistor The simulation analysis contains only the power circuits [6].

Scheme of the inverter is shown in Fig. 6. This inverter is powered from the DC voltage $V_{1}=25 \mathrm{~V}$, where the $\mathrm{R} 5$ represents the internal resistance of the input voltage source. To the input side of converter is connected filter capacitance with the parasitic serial resistance. Transistors are controlled by the generator of voltage impulses with desired frequency and shape. Used transistor is IRFI540N. Clamps PIN 1, PIN 2, +V1 are connected to transformer circuit.

Transformer is created by simplified substitution circuit, which is shown in Fig. 7. The network contains the inductors $\mathrm{L}_{2}, \mathrm{~L}_{3}, \mathrm{~L}_{4}$,

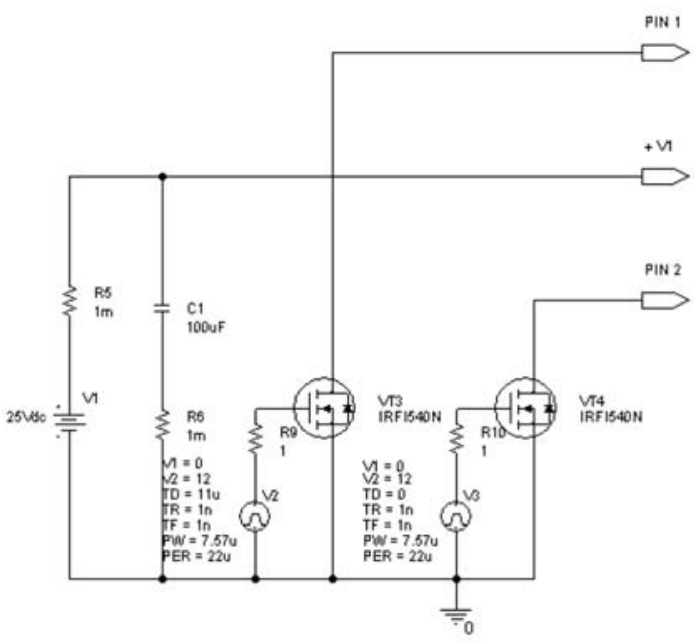

Fig. 6 Scheme of the inverter for simulation analysis

$\mathrm{L}_{5}$, among which is ideal magnetic coupling. The values of these inductances are specified by the ratio of transformer. Inductances $\mathrm{L}_{6}, \mathrm{~L}_{7}, \mathrm{~L}_{8}, \mathrm{~L}_{9}$ are representing the dissipation inductances of the transformer and the effective resistances of winding are $R_{3}, R_{4}$ a $R_{7}, R_{8}$. The input pins PIN 1, PIN 2, + V1 are connected to output of inverter and PIN 3, PIN 4 and PIN 5 to the input of synchronous rectifier.

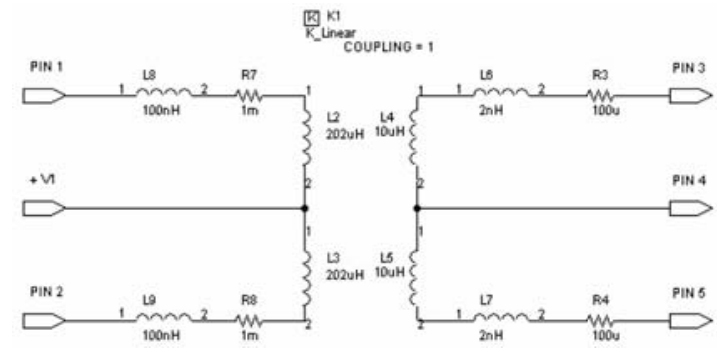

Fig. 7 Scheme of the power transformer used for simulation analysis

Fig. 8 shows the circuit of diode rectifier. Used transistors IRF1310NS are not controlled. With this setting, with zero gate signal, the internal diode of the structure conducts the current. The transistor acts as a diode. The inductor $\mathrm{L}$ presents as output filter of the rectifier. Capacitor $\mathrm{C}_{2}$ is output capacitance with serial parasitic resistance $R_{1}$. Resistors $R_{11}$ and $R_{12}$ are used for elimination of the convergence of the computation in simulation. Input clamps PIN 3, PIN 4 and PIN 5 of the rectifier simulation analysis are connected to output pins of the circuit of the transformer.

Circuit of the synchronous rectifier is shown in Fig. 8. The transistors IRF1310NS are also used. The drive voltage supplies are set on $10 \mathrm{~V}$. Switching of these transistors is synchronous to the switching of inverter. Input pins PIN 3, PIN 4, PIN 5 are connected to the output of the network of the transformer. 


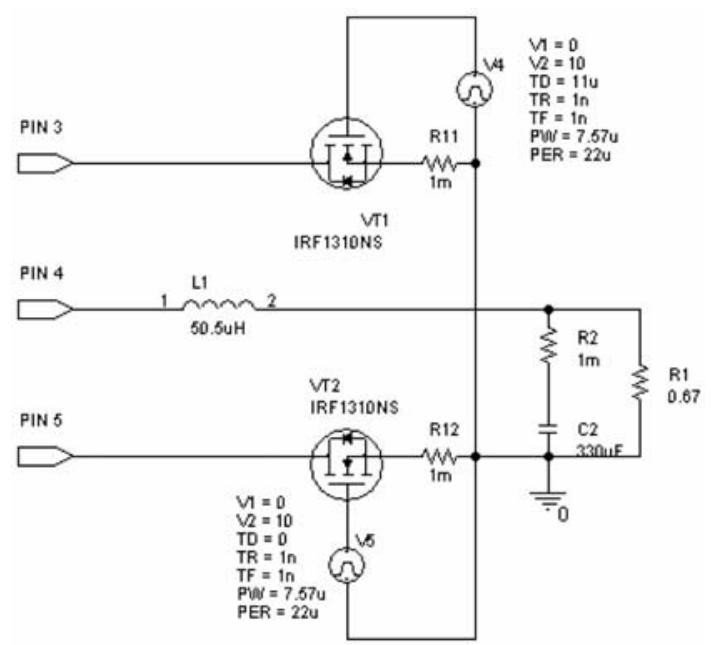

Fig. 8 Scheme of the diode and synchronous rectifier used for simulation analysis

Simulation analysis was done with input voltage of $V_{1}=25 \mathrm{~V}$. Frequency of the impulse generator of $45 \mathrm{kHz}$. Changing of the current was achieved by changing of the output resistance $\mathrm{R}_{1}$ and the demanded value of the output voltage of $3.5 \mathrm{~V}$ with the right value of duty cycle of the PWM driving signals for inverter and rectifier.

Fig. 9 shows the comparison of the efficiency of the switching converter with diode and synchronous rectifier. It is easy to see that the efficiency of synchronous rectifier is higher. The largest efficiency, $74.613 \%$ is with output current $7.44 \mathrm{~A}$. With the nominal output current $18 \mathrm{~A}$ it is $74.3 \%$. The biggest difference of the efficiency is when the output current is $3.333 \mathrm{~A}$.

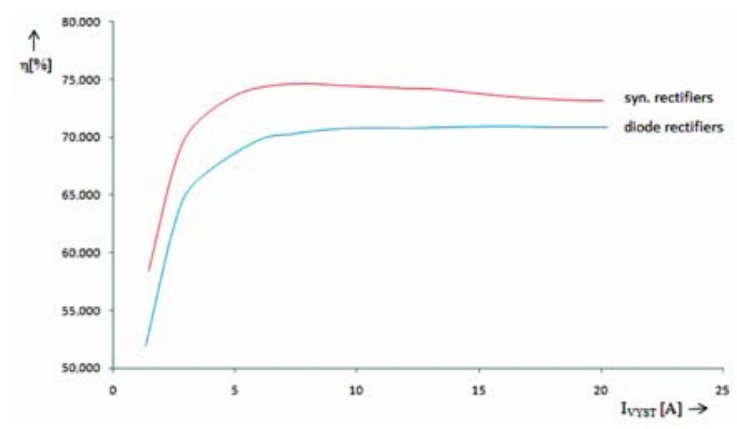

Fig. 9 Efficiency characteristics

In Fig. 10 is shown the comparison of power dissipation on the transistor with and without drive, where it acts as diode. With the increasing output current, the power losses increased too.

In Figs. 11 and 12 are waveforms of the electrical values of the circuit's simulation analysis. It was done with input voltage $V_{1}=$

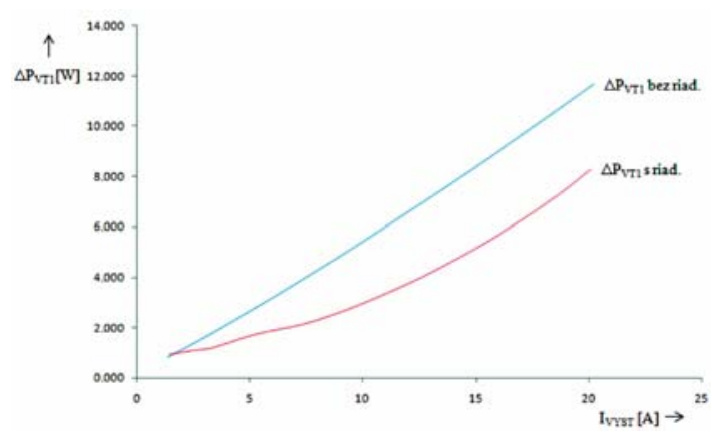

Fig. 10 Comparison of the losses on transistor with and without control

$=25 \mathrm{~V}$, input current $I_{I N}=0.99$ A, output voltage $V_{\text {OUT }}=3.5 \mathrm{~V}$ and output current $I_{\text {OUT }}=5.22 \mathrm{~A}$.

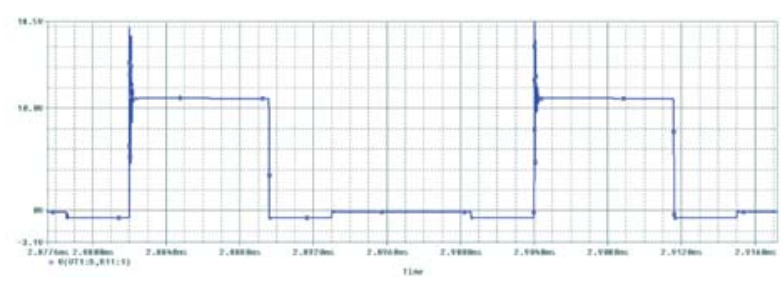

Fig. 11 Waveform of the voltage UDS on the transistor VT1

From the waveforms of the voltage on transistor VT1 it is easy to see that used control of synchronous rectifier is not so efficient, because the transistor was not open at full time until the current dissolves. The inner diode is applied for a long time. With better control, the power losses on the transistor will decrease.

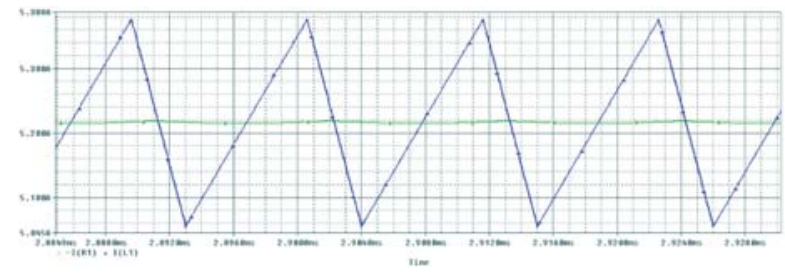

Fig. 12 Waveform of the output current and the current through filter inductor

The ripple of output current is lower than $5 \%$ of its average value (Fig. 12). This satisfied the condition for the source used for electroplating.

\section{Switching Power Supply Experimental Verification}

In experimental verification we used the push-pull inverter connection (Fig. 13), which uses the entire hysteresis core. Converter is able to work with $50 \%$ of the pro-open. Control is ensured with 
the UCC28083 circuit for both inverter and synchronous rectifier Parameters for designing switching power supply are as follows:

- input voltage 20-36 VDC

- output voltage $3.5 \mathrm{VDC}$

- output current $18 \mathrm{~A}$

- switching frequency $45 \mathrm{kHz}$

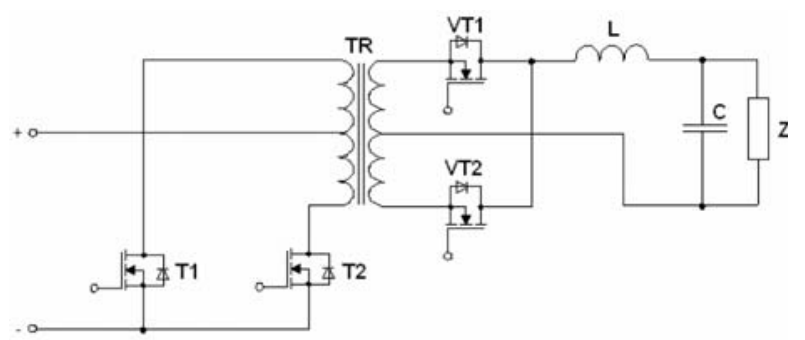

Fig. 13 PUSH-PULL converter with synchronous rectifier

The waveforms are captured for input voltage $U_{I N}=25 \mathrm{~V}$, input current $I_{I N}=0.99 \mathrm{~A}$ and output voltage $U_{O U T}=3.5 \mathrm{~V}$, output current $I_{\text {OUT }}=5.22 \mathrm{~A}$. The system uses control, which is shown in Fig. 7a. In the waveform of transistor voltage VT1 (Fig. 15) of the synchronous rectifier we can see that the regulation is not fully effective, because the transistor isn't switched throughout the period when current is conducted through a given branch. This fact is caused by the body diode of transistor. Therefore, we used the following synchronous rectifier control circuit, which is in Fig. 14b. The waveform of transistor voltage with this control is shown in Fig. 16.

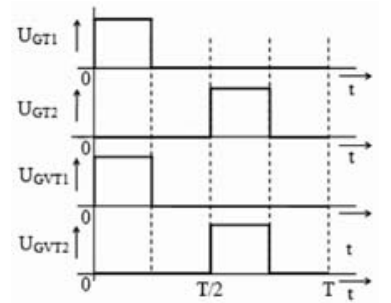

a) Control 1

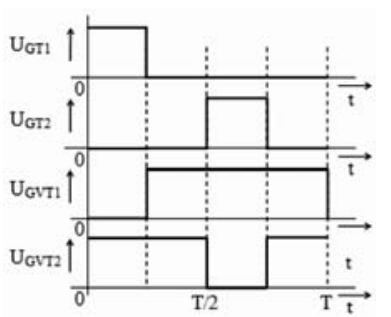

b) Control 2
Fig. 14 Switching diagram of control signals

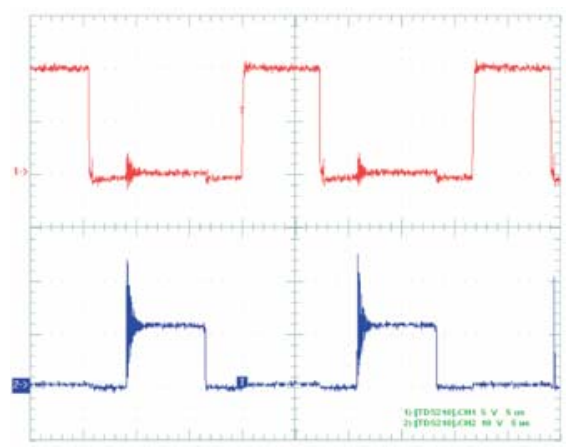

Fig. 15 Waveform of transistor voltage VTI $\left(1-U_{G}, 2-U_{D S}\right)$

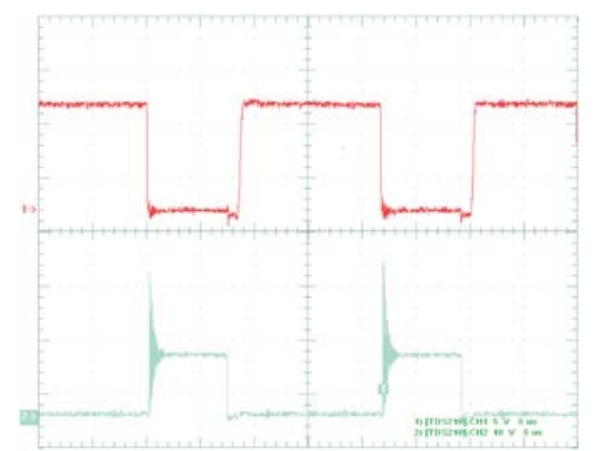

Fig. 16 Waveform of transistor voltage VT1 (1-U, $\left.2-U_{D S}\right)$

Measurement of efficiency was made at the input voltage $U_{I N}=25 \mathrm{~V}$. The efficiency was measured without transistors drivers, when only the body diodes of transistors were applied. To measure the diode rectifier we set the maximum output current on $8 \mathrm{~A}$, because the transistor didn't have additional cooling and could be destroyed. Measurement results are in Fig. 17. The transistor efficiency is much better when using drivers. This fact is well visible from characteristics. Efficiency for small output current decreases, which is caused by increasing of mutual ratio between driving the switching losses due to transferred power source. With increasing output current above 8A, efficiency decreases too, because of increasing the conduction losses. In the alternative control, efficiency increased because the body diode wasn't applied.

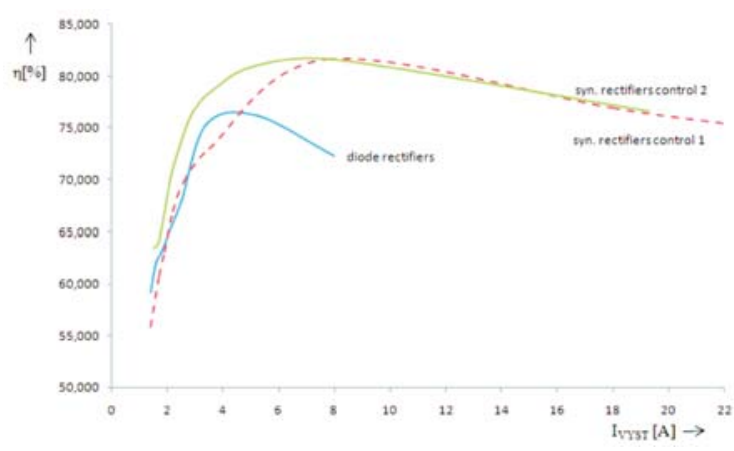

Fig. 17 Efficiency characteristics

In Fig. 18, we can see thermal image of uncontrolled transistors rectifier. Thermal images of the rectifier with the first control are in Fig. 18b and those with the second one in Fig. 18c. Images were made at an output current $5.2 \mathrm{~A}$. In the case of the second control, transistor has the lowest temperature because of there are no losses on the body diode.

\section{Conclusion}

The main goal of this experiment (simulation analysis) was to increase efficiency of switching power supply using a synchronous 


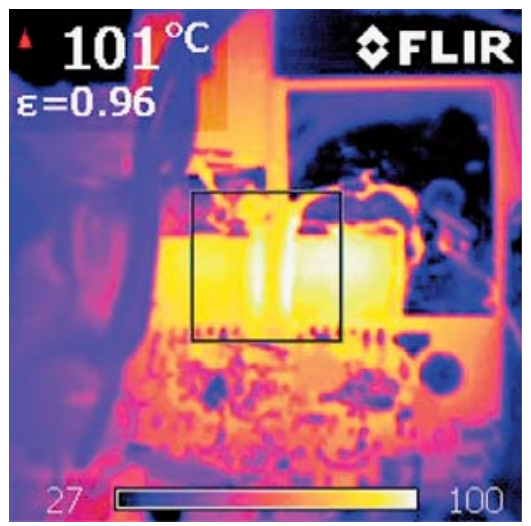

a) Diode rectifier

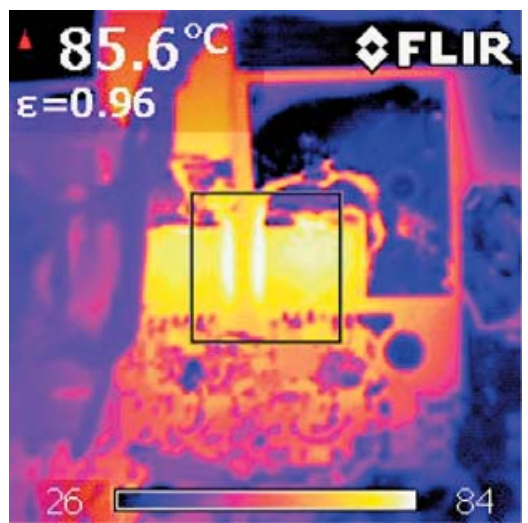

b) Synchronous rectifier - control 1

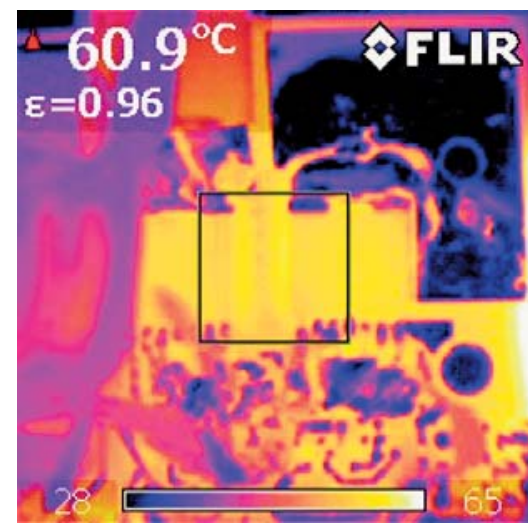

c) Synchronous rectifier - control 2

Fig. 18 Thermal images of transistors VTI and VT2

rectifier. The efficiency of switching power supply at output current $18 \mathrm{~A}$ is $76.9 \%$ in the case of "control 1 ". The maximum efficiency is $81.636 \%$ at an output current $7.8 \mathrm{~A}$. In the second type synchronous rectifier control (control 2) the efficiency is $80 \%$ (output current is $18 \mathrm{~A}$ ). The maximum efficiency is $81.591 \%$ at output current 8.1A. Greater efficiency could be achieved using a suitable transistor synchronous rectifier, where static and dynamic parameters of the transistor and also its body diode are very important: the smallest internal resistance in the conductive state $R_{D S(o n)}$, body diode threshold voltage and dynamic resistance $R_{D}$. Used topology for this synchronous rectifier is suitable for high currents, because the current always flows through only one transistor to reduce the losses. The results of this experiment will help in the design of the source with output current of thousands of amps for electroplating industry.

\section{Acknowledgement}

The authors wish to thank for the financial support of the operational program of R \& D Centre of Excellence of power electronic systems and components for material OPVaV-2008/2.1/01SORO, ITMS 26220120003 funded by the European Regional Development Fund (ERFD) and VMSP-P-0085-09, APVV -053507.

\section{References}

[1] http://www.odbornecasopisy.cz, 2004

[2] STOIC, G., NGUIEN, C.: MOSFET Synchronous Rectifiers for Isolated, Board-Mounted DC-DC Converters. Proc. of Conference INTELEC'2000 Arizona, USA, 2000

[3] HARGAS, L., HRIANKA, M., LAKATOS, J., KONIAR, D.: Heat Fields Modelling and Verification of Electronic Parts of Mechatronics Systems, Metalurgija - Metallurgy, Vol. 49, No. 2, 2010, pp. 268-272

[4] LEE-HUN, KIM, KWANG-SU, CHUN, CHUNG-YUEN, WON, SOO-SEOK, KIM, SE-WAN, CHOI.: An Improved Scheme for High-Efficiency Push-Pull Converter Using Single Winding Self-Driven Synchronous Rectification, Industrial Electronics Society, 2004

[5] MOHAN, N., UNDELAND, T.M, ROBBINS, W.P.: Power Electronics: Converters, Applications and Design. Johny Wiley \& Sons, New York, 1989

[6] OrCAD Pspice A/D: User's Guide, Beaverton, 1998. 